\title{
An Investigation on Improvements to Ultrasound Reactor Configuration for Extraction of Phytochemicals in Industrial Scale
}

\author{
Tarık Uçar \\ Department of Agricultural Biotechnology, Tekirdag Namik Kemal University, \\ Environics Applied Sciences Inc., Tekirdag, Turkey \\ Aziz Kubilay Ovacıklı \\ Department of Computer Science, Istanbul Rumeli University, Istanbul, Turkey \\ Environics Applied Sciences Inc., Tekirdag, Turkey
}

\begin{abstract}
Industrial attentiveness and demand for natural bioactive compounds are rising continuously, regarding their growing commercial values in nutrition, pharmaceuticals, cosmetics and many other industries. The progress in ultrasound technology has spread the use of ultrasonication on a broad field of application areas, including extraction of bio-materials from plant sources. Ultrasound-assisted extraction is a powerful alternative to conventional techniques, in terms of extracting bio-compounds from variable kinds of matrices, higher efficiency, reduced extraction time, toxic-free operation, lower energy demand, lesser water consumption and better extract quality. Existing research pointed out that the reactor configuration is vital for maximizing the efficacy of extraction process, however basic reactor configurations that were mainly used in the literature may not be feasibly adapted to full-scale industrial applications. In this study, an investigation on possible improvements and modifications to existing reactor designs are discussed, such as detecting the optimum frequency range of ultrasound transmission depending on the material rheology and composition, possible modifications in beam-forming by means of frequency modulation and finally transmitting the waves in combination of different frequencies.
\end{abstract}

Keywords: ultrasound, frequency, extraction, phytochemical

\section{Introduction}

Phyto means plant in Greek and phytochemicals are natural bio-molecules that are synthesized by plants for protection against external threats such as pollution, stress, 
pathogens, UV exposure, etc. (Saxena et. al., 2013) There are more than 4.000 phytochemicals identified today that can be encountered in fruits and vegetables, grains, legumes, herbs and even fungi (Saxena et. al., 2013).

Phytochemicals are highly valuable chemical compounds as recent studies uncovered that, even if they are not essential nutrients for humans, they provide well-being by showing antioxidant properties, biocidal effects, modulating detoxification enzymes, stimulating immune system, regulating hormone system and anticancer properties (Saxena et. al., 2013). It is reported that individuals who regularly consume fruits and vegetables, carry \%50 less risk of cancer according to their opposite counterparts and this benefit attributes to the consumption of phytochemicals (Boyer \& Liu, 2004). Besides nutrition, phytochemicals also have application areas in industrial processes, such as food, cosmetics and pharmaceuticals. $\% 90$ of the extracted tannin today is used in tanning process of leather industry (Sivakumar, 2007).

Extraction is a process that we encounter in our everyday lives and it dates back to ancient times (Panda \& Manickam, 2009). Tea and coffee are both extracts which we obtain via thermal extraction methods, everyday. Basically, extraction can be defined as a separation process used for separating a substance of interest from a matrix. Undoubtedly, extraction is an indispensable fragment of industrial manufacturing today, especially in food production, cosmetics, nutraceuticals and pharmaceuticals (Panda \& Manickam, 2009).

Conventional extraction techniques, generally referred to as thermal methods, are widely used for industrial purposes. Conventional methods that are in use for extraction of vegetal materials can be listed as follows (Vinatoru, 2001);

- Solvent Extraction

- infusion

- enfleurage

- maceration

- percolation

- extraction with hot fat

- Distillation

- water and steam distillation

- water steam distillation

- distillation of essential oils

- Cold Compression

Even though above given extraction methods have been applied successfully for years, two main driving forces have led the industry to search more advanced extraction techniques. First is the globally increasing energy costs. A faster method which can complete the extraction process in less time, also requires lower energy consumption than conventional techniques, will surely lower the production costs of the industry, 
significantly. On economic aspect, installing conventional extraction units approximately requires $\% 50$ of the investment of a new production plant, and they represent $\% 70$ of the total energy consumption of a food plant (Chemat et. al., 2017). With these numbers in mind, any advancement in extraction technology means a lot to manufacturing industry. The second driving force is the increased consumer awareness. Consuming safer and more nutritive products (Aneja et. al., 2014) has been gaining popularity worldwide that manufacturers need to take notice. An innovative extraction technique which can eliminate the usage of toxic chemicals, operates with lower temperatures for obtaining heat-sensitive biomolecules and eventually provides higher extract quality (Chemat et. al., 2017), would probably better meet the market demands.

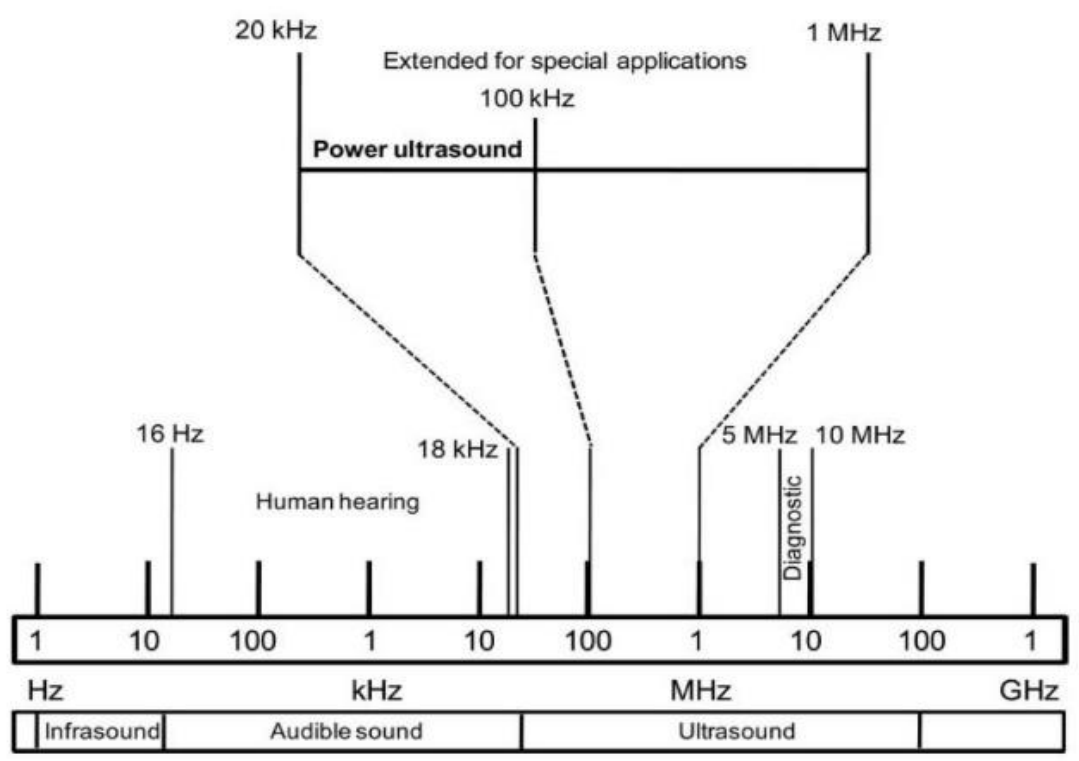

Ultrasound is basically defined as acoustic sound waves (emitted by probes), over human hearing range ( $>20 \mathrm{kHz}$, see Figure 1$)$, propagating through a medium, creating mechanical vibrations at a certain frequency. These waves can either be transmitted in a pulse-echo mode or continuous wave propagation, causing different effects in the bulk.

Figure 1. Frequency ranges of sound (Rastogi, 2011)

Ultrasound technology have found itself several practises in food manufacturing, as can be seen in Table. 1

\begin{tabular}{|l|l|}
\hline Mechanical Actions & Chemical and Biological Actions \\
\hline crystallization & biocidal effects \\
\hline
\end{tabular}




\begin{tabular}{|l|l|}
\hline degassing & waste treatment \\
\hline foam destruction & $\begin{array}{l}\text { regulation of growth of living } \\
\text { cells }\end{array}$ \\
\hline extraction of flavourings & modification of enzyme activities \\
\hline filtration & sterilization of equipments \\
\hline drying & \\
\hline freezing & \\
\hline mixing & \\
\hline tenderization of meat & \\
\hline
\end{tabular}

Table 1. Application Areas of Ultrasound in Food Industry. (Mason et. al., 2005)

Applying ultrasound in extraction process is called ultrasound assisted extraction (UAE) (Vinatoru et. al., 2017). Extraction technology that uses ultrasound can be operated with lower temperatures than conventional thermal methods, hence degradation of heatsensitive molecules can be avoided (Panda \& Manickam, 2009). It is also considered as a green technology as green and lesser amounts of solvents can be used in place of toxic chemicals (Panda \& Manickam, 2009). Besides giving the chance of improvements on safety and quality of foods, ultrasound technology also gives the opportunity to develop and design new products (Patist \& Bates, 2008).

Besides all its beneficial properties, unlike from other innovative technologies in the field, ultrasound reactors to be used in extraction are not off-the-shelf products and need to be engineered application specific (Patist \& Bates, 2008; Patist \& Bates, 2008 ).

The presented article focuses on the ultrasound assisted extraction (UAE) of phytochemicals and possible applications on industrial scale by incorporating signal processing and analysis methods to optimize the extraction process. Difficulties in scaling the ultrasound treatment to an industrial application has been studied thoroughly (Tiwari, 2015) and problems associated with it have been noted. The following sections will discuss the induced effects of ultrasound applications in extraction, the variables influencing efficiency in terms of extracted yield and possible improvements that can be achieved by applying detection methods through the extraction process to identify the optimum values for the parameters.

\section{Background}

The principle behind UAE is the cavitation induced by the high power, low frequency ultrasound applied on a matrix, which is also referred as macrosonics. In other words, ultrasound applied with relatively high power (low frequency range $20-500 \mathrm{kHz}$, high 
intensity greater than $1 \mathrm{~W} / \mathrm{cm} 2$ ) have an impact especially on the chemical composition of food through cavitation (Awad et al., 2012) which enables the extraction of compounds that can later be used in various applications mentioned in the previous section. Cavitation (referring to as transient and unstable acoustic cavitation hereafter) by creating heat, pressure and turbulence (Patist, \& Bates, 2008) cause cell disruption enabling the penetration of the solvent that will then let extraction of the target ingredient, until an equilibrium in the bulk is reached. Investigation of equations governing this phenomenon is out of the scope of the presented work, a comprehensive study will be done later on a specific type of food and its ingredients. Numerous studies can be found describing the basics of this well-known yet difficult to control physical event (Chandrapala et al., 2012). For instance, linear equations describing the propagation of ultrasonic waves disregarding compressibility were given, whereas the nonlinearities occurring especially in resonant cases (observed in high power ultrasound) were also studied (Laborde et al., 1998). Relating ultrasonic parameters such as wavelength to reactor geometry, resonant diameter to frequency (inversely proportional) already gives some hints on maximizing the cavities collapsing on the surface thus extracted yield (Patist, \& Bates, 2008). Here, it was noted that the power dissipation would be the main concern in industrial scale applications. In another study, modelling of the acoustic pressure field (Moholkar et al., 2000), mathematical modelling and numerical simulations on the spatial distribution of the cavitation were investigated. Nevertheless, scaling the experimental setup up to an industrial production level still possesses problems when it comes to the generalization of the geometry, regression models and optimum process parameters, independent of the application and component of interest.

To provide a more detailed background of the previous studies, entire literature consists of empirical studies as the (transient) cavitation phenomenon is highly nonlinear in nature (Laborde et al., 1998). The basic outcome is that each experiment aims determining optimum conditions and parameters of a specific application, be it extraction of particular ingredients, decontamination or characterizing the physical properties of a given matrix. What is common in all these studies is the factors affecting the UAE efficiency, namely the reactor type (ultrasonic bath or probe/horn type) as illustrated in Figure 2, frequency of the emitted acoustic waves, applied power or intensity, duration, process temperature, solvent type, ratio and properties (Tiwari, 2015) and matrix size (Vinatoru et. al., 2001). In addition to these, probe design and geometry, utilizing multiple ultrasound probes to enhance cavitational effects can also be listed (Bilek \& Turantaş., 2013; Awad et al., 2012), as well as ultrasound probe operating at continuous/pulsed mode focused on the sample to produce cavitation concentrated on a fixed area. 


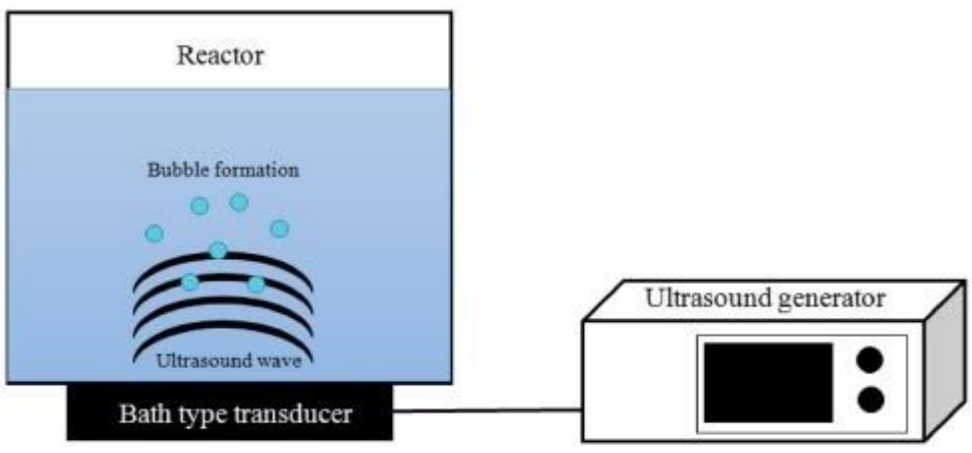

a. Ultrasonic bath type emitter.

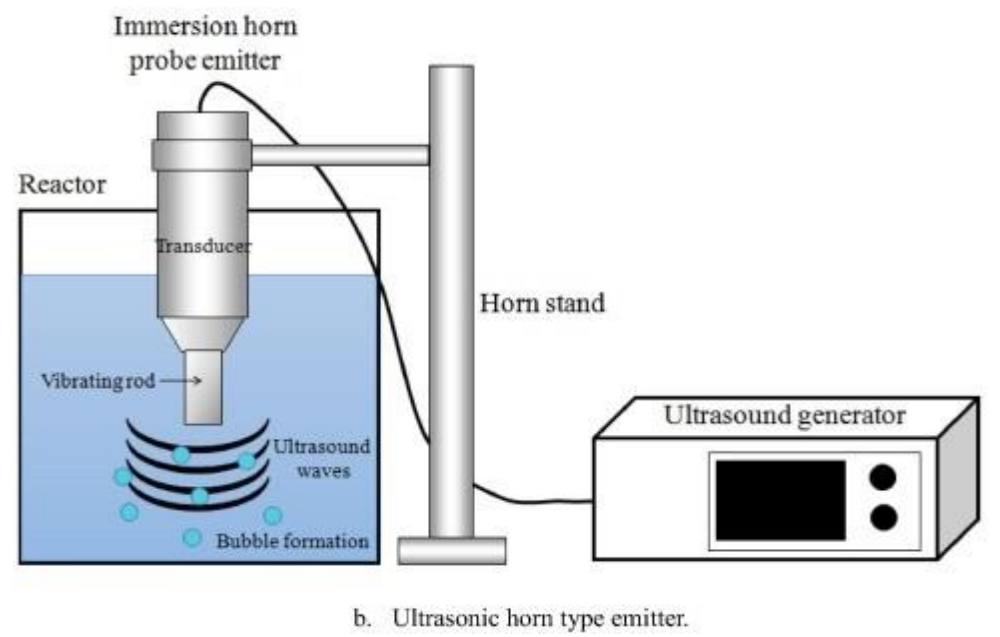

Figure 2. Ultrasonic Reactor Types (Thokchom et. al., 2015)

\section{Discussion}

Cavitation phenomenon can be investigated by frequency analysis (Knorr et al., 2004) with its effects on extraction capabilities, to aid identifying the patterns and specific frequencies characterizing the constitution and physicochemical properties of food. Optimization of extraction through design of experiments and data-driven models for improvement of UAE on a specific phytochemical to provide insight to the possibilities of developing industrial scale devices will be the concern of research following the presented work here. In addition to that, detecting cavitation (and its intensity) alone with an improved design of ultrasonic transducers may further enhance such possibilities (Bornmann et al., 2014). That will in return lead to a study on harmonic content of the reflected echoes or related variables that may lead to identifying process behaviour for a specific application. For instance, detection of impulsive occurrences (since collapsing cavities can be considered as impulsive events) within a given duration 
with time domain approaches might lead to a better understanding of when and at what intensity cavities collapse given a specific phytochemical.

Collapsing bubbles are very short duration and very intense events which can be considered as impulses that could be detected as transients in acoustic measurements. Signal processing tools for the analysis of data collected during UAE of several species and building data driven models on the processed information, making use of multidisciplinary collaboration and knowledge are expected to provide novel approaches and scientific contribution to the field. To exemplify, high speed jets arising due to the hydrodynamic cavitation are known to induce erosion even on the surface of the pump vanes, creating sudden malfunction in industrial processes causing unplanned downtime in the production. Some attempts had been made to understand and detect this phenomena to design an early detection system in different fields (Stopa et al., 2013; Čdina, 2003). Such approaches and studies could be beneficial to better understand the principles of cavitation in food processing.

The ultimate purpose here is to establish online monitoring of the extraction process by continuously measuring the parameters such as temperature, $\mathrm{pH}$, acoustic intensity, acoustic field inducing transient cavitation in high power ultrasound to associate these with the mechanical properties of phytochemicals such as attenuation coefficient and acoustic impedance (Awad et al., 2012) and the solvent such as density, viscosity, homogeneity (Vinatoru et al., 2017; Vilkhu et al., 2008). In such an attempt, the results of the facts that the ultrasound waves are absorbed, scattered, reflected and finally induced cavitation which all cause certain amount of energy reduction will be taken into account. All these effects must be considered when adjusting the applied power to a specific matrix to extract the compound of interest with max. yield (Tiwari, 2015). Calorimetry (assuming that the temperature of the entire bath remains approximately constant or varies within a small range) can be used to get an estimate of the applied power. However, such assumptions might only be valid in laboratory-scale applications. To reach a better understanding of the necessities concerning an industrial scale reactor, specific analysis tools and detection methods will be utilized.

To integrate the bubble dynamics (Moholkar et al., 2000; Gogate \& Pandit, 2004), a comprehensive discussion on sonochemical reactors and optimization of operating parameters (intensity, frequency, physicochemical properties of the medium) must be accomplished. Producing standard experimental setup does not sound reasonable and thus generalization of the design parameters and experiments are not realistic. Depending on the application, optimum parameters (affecting each other) must be found primarily with empirical approaches. Data derived from cavitational yield and other measurable parameters may reveal correlations between them. Concept of multiple transducers with an optimum geometric configuration leading to modulation of waves at desired frequencies may enhance the acoustic properties of the reactor. Viscosity of the solvent and free radical formation (due to the collapse of cavities in solvent (Soria \& 
Villamiel, 2010) are other concerns that must be dealt with when it comes to the estimation of the optimum settings.

\section{Conclusion}

Ultrasound Assisted Extraction of phytochemicals at its current stage was investigated and it was noted that using the process data that is produced by a nonlinear system, fitting to 2nd order polynomials, attempting to design regression models and being highly determined by the physical and biochemical properties of the material may produce conclusive results in small-scale applications but will not be applicable in industrial production plants. There is still room for improvement especially on the data analysis and processing part of the parameters, such as identifying the relation between vapor pressure within the bubbles and intensity of collapsing bubbles, surface tension of the bulk and threshold of transient cavitation (Tiwari, 2015), as well as the solvent viscosity.

In addition to what has been mentioned so far, multi-frequency systems may help to understand the combined effects of ultrasound transmitted in different frequencies. By doing so, data corresponding to extracted compounds may be related to modulation and frequency bands. Sonication duration must be adjusted in such a way to maximize the extract while hindering the occurrence of free radicals, or by making use of other methods (gas injection or mixtures of solutants must be preferred). Applying signal processing techniques on UAE will be the concern of further research following the presented study.

\section{References}

[1] Thokchom, B., Pandit, A. B., Qiu, P., Park, B., Choi, J., \& Khim, J. (2015). A review on sonoelectrochemical technology as an upcoming alternative for pollutant degradation. Ultrasonics Sonochemistry, 27, 210-234.

[2] Vinatoru, M. (2001). An overview of the ultrasonically assisted extraction of bioactive principles from herbs. Ultrasonics sonochemistry, 8(3), 303-313.

[3] Mason, T. J., Riera, E., Vercet, A., \& Lopez-Buesa, P. (2005). Application of ultrasound. In Emerging technologies for food processing (pp. 323-351). Academic Press.

[4] Saxena, M., Saxena, J., Nema, R., Singh, D., \& Gupta, A. (2013). Phytochemistry of medicinal plants. Journal of pharmacognosy and phytochemistry, 1(6).

[5] Bilek, S. E., \& Turantaș, F. (2013). Decontamination efficiency of high power ultrasound in the fruit and vegetable industry, a review. International journal of food microbiology, 166(1), 155-162.

[6] Chemat, F., Rombaut, N., Sicaire, A. G., Meullemiestre, A., Fabiano-Tixier, A. S., \& Abert-Vian, M. (2017). Ultrasound assisted extraction of food and natural 
products. Mechanisms, techniques, combinations, protocols and applications. A review. Ultrasonics sonochemistry, 34, 540-560.

[7] Tiwari, B. K. (2015). Ultrasound: A clean, green extraction technology. TrAC Trends in Analytical Chemistry, 71, 100-109.

[8] Soria, A. C., \& Villamiel, M. (2010). Effect of ultrasound on the technological properties and bioactivity of food: a review. Trends in food science \& technology, 21(7), 323-331.

[9] Panda, D., \& Manickam, S. (2019). Cavitation technology-The future of greener extraction method: A review on the extraction of natural products and process intensification mechanism and perspectives. Applied Sciences, 9(4), 766.

[10] Sivakumar, V., Verma, V. R., Rao, P. G., \& Swaminathan, G. (2007). Studies on the use of power ultrasound in solid-liquid myrobalan extraction process. Journal of Cleaner Production, 15(18), 1813-1818.

[11] Aneja, K. R., Dhiman, R., Aggarwal, N. K., \& Aneja, A. (2014). Emerging preservation techniques for controlling spoilage and pathogenic microorganisms in fruit juices. International Journal of Microbiology, 2014.

[12] Boyer, J., \& Liu, R. H. (2004). Apple phytochemicals and their health benefits. Nutrition journal, 3(1), 5.

[13] Rastogi, N. K. (2011). Opportunities and challenges in application of ultrasound in food processing. Critical reviews in food science and nutrition, 51(8), 705-722.

[14] Stopa, M. M., Cardoso Filho, B. J., \& Martinez, C. B. (2013). Incipient detection of cavitation phenomenon in centrifugal pumps. IEEE Transactions on industry applications, 50(1), 120-126.

[15] Patist, A., \& Bates, D. (2008). Ultrasonic innovations in the food industry: From the laboratory to commercial production. Innovative food science \& emerging technologies, 9(2), 147-154.

[16] Knorr, D., Zenker, M., Heinz, V., \& Lee, D. U. (2004). Applications and potential of ultrasonics in food processing. Trends in Food Science \& Technology, 15(5), 261-266.

[17] Awad, T. S., Moharram, H. A., Shaltout, O. E., Asker, D., \& Youssef, M. M. (2012). Applications of ultrasound in analysis, processing and quality control of food: A review. Food research international, 48(2), 410-427.

[18] Bornmann, P., Hemsel, T., Sextro, W., Memoli, G., Hodnett, M., \& Zeqiri, B. (2014, September). Self-sensing ultrasound transducer for cavitation 
detection. In 2014 IEEE International Ultrasonics Symposium (pp. 663-666). IEEE.

[19] Laborde, J. L., Bouyer, C., Caltagirone, J. P., \& Gérard, A. (1998). Acoustic cavitation field prediction at low and high frequency ultrasounds. Ultrasonics, 36(1-5), 581-587.

[20] Gogate, P. R., \& Pandit, A. B. (2004). Sonochemical reactors: scale up aspects. Ultrasonics Sonochemistry, 11(3-4), 105-117.

[21] Čdina, M. (2003). Detection of cavitation phenomenon in a centrifugal pump using audible sound. Mechanical systems and signal processing, 17(6), 13351347.

[22] Moholkar, V. S., Rekveld, S., \& Warmoeskerken, M. M. (2000). Modeling of the acoustic pressure fields and the distribution of the cavitation phenomena in a dual frequency sonic processor. Ultrasonics, 38(1-8), 666-670.

[23] Vilkhu, K., Mawson, R., Simons, L., \& Bates, D. (2008). Applications and opportunities for ultrasound assisted extraction in the food industry-A review. Innovative Food Science \& Emerging Technologies, 9(2), 161-169.

[24] Patist, A., \& Bates, D. (2008). Ultrasonic innovations in the food industry: From the laboratory to commercial production. Innovative food science \& emerging technologies, 9(2), 147-154.

[25] Chandrapala, J., Oliver, C., Kentish, S., \& Ashokkumar, M. (2012). Ultrasonics in food processing. Ultrasonics sonochemistry, 19(5), 975-983.

[26] Vinatoru, M., Mason, T. J., \& Calinescu, I. (2017). Ultrasonically assisted extraction (UAE) and microwave assisted extraction (MAE) of functional compounds from plant materials. TrAC Trends in Analytical Chemistry, 97, 159-178. 